\title{
El nexo migración-desarrollo y la economía política de la vivienda en América Latina
}

Gisela P. Zapata*

RESUMEN

En los últimos años, varios gobiernos de América Latina han promovido la noción de que los migrantes pueden actuar como agentes de desarrollo, principalmente mediante la canalización de remesas hacia sectores específicos como los de vivienda y financiero. Los estudios desarrollados hasta el momento aún no han expuesto cómo estos hechos están reconfigurando la economía política de la vivienda en la región. Con base en datos empíricos recogidos en ambas puntas de la cadena migratoria Colombia-Reino Unido, este artículo sostiene que estos esfuerzos tienen como objetivo institucionalizar los flujos de remesas y las actividades socioeconómicas transnacionales que de ellas se derivan. Estos esfuerzos están fundamentados en el reposicionamiento de la vivienda como un bien de consumo a un bien de inversión y motor del crecimiento económico, al igual que del sector financiero como el principal medio de los hogares para acceder a vivienda pública y privada, y a otros servicios básicos. Estas acciones han tenido lugar en el contexto de un proceso más amplio de financialización de la agenda global de pobreza durante las últimas tres décadas.

Palabras clave: migración transnacional, remesas, desarrollo, vivienda, financialización, Colombia.

* Doctora en Geografia Humana, Newcastle University (Reino Unido). Investigadora de posdoctorado, Centro de Desenvolvimento e Planejamento Regional (CEDEPLAR), Universidad Federal de Minas Gerais (Brasil). gpzapata@cedeplar.ufmg.br/gpzapata@gmail.com

Recibido: 10 de abril de 2015 / Modificado: 20 de mayo de 2015 / Aceptado: 20 de enero de 2016 Para citar este artículo

Zapata, G. P. (2016). El nexo migración-desarrollo y la economía política de la vivienda en América Latina. OAsIs, 23, 123-144. DOI: http://dx.doi.org/10.18601/16577558.n23.07 
The migration-development nexus and the political economy of housing in Latin America

\section{ABSTRACT}

In recent years, various Latin American governments have attempted to render migrants as agents of development, mainly by channeling remittances to specific sectors such as housing and finance. The existing literature has yet to articulate how these new developments are reconfiguring the political economy of housing in the region. Drawing on empirical data collected at both ends of the Colombia-uk migration network, this paper argues that these efforts aim to institutionalise remittance flows and their associated transnational socio-economic activities. They are underpinned by the repositioning of housing from a consumption to an investment item, and as a driver of economic growth; as well the positioning of the financial sector as the main means for households to access public and private housing, and other basic services. These developments have taken place in the context of a broader process of financialisation of the global poverty agenda in the last three decades.

Key words: Transnational migration, remittances, development, housing, financing, Colombia.

\section{INTRODUCCIÓN}

Siguiendo el ejemplo del Banco Mundial y otras instituciones financieras internacionales (IFI), en los últimos años varios gobiernos de América Latina han tratado de reincorporar a sus migrantes a la política nacional para convertirlos en agentes de desarrollo. Esto lo han hecho a través de una variedad de iniciativas de cuño político, social, cultural y económico, tales como la ampliación de los derechos políticos de los migrantes, el patrocinio de asociaciones formales de migrantes, la creación de centros culturales en el exterior, la reducción del costo de envío de remesas y, principalmente, la canalización de remesas para proyectos de desarrollo y de inversión "productivos". Los llamados programas de migración para el desarrollo promueven la idea de que los migrantes y sus remesas pueden actuar como impulsores del desarrollo económico en sus países de origen $^{1}$ (Faist, 2008; Portes, 2003). A pesar de los actuales esfuerzos de los gobiernos para incorporar a los trabajadores migrantes a sus políticas de desarrollo nacional a través de la canalización de las remesas a sectores específicos como los de vivienda y financiero, los estudios desarrollados hasta el momento aún no han expuesto cómo estos nuevos hechos están reconfigurando la economía política de la vivienda en América Latina.

Este artículo busca llenar este vacío por medio de una revisión de las tendencias en materia de políticas y prácticas para el desarrollo

Esta idea se ha tornado tan importante entre las agencias internacionales, la academia y los círculos políticos, que el Reporte de Desarrollo Humano de las Naciones Unidas de 2009 se dedicó a este tema (UNDP, 2010). 
y la financialización del sector de la vivienda en América Latina, con especial referencia a los esfuerzos del Gobierno colombiano para convertir a los migrantes en agentes de desarrollo, haciéndolos objeto de una nueva política de vivienda. Esto es importante, dado que existe un conocimiento limitado sobre el uso y la producción de la vivienda y su financiamiento en los países en desarrollo (Datta y Jones, 2001), y hay una necesidad urgente de "explorar sistemáticamente el entorno político en el cual están incorporados los esfuerzos para establecer un vínculo entre la migración y el desarrollo" (Geiger y Pécoud, 2013, p. 373).

El artículo sostiene que la elección de los sectores de vivienda y financiero para la incorporación de los migrantes colombianos a la política nacional no es una coincidencia, sino más bien, un esfuerzo deliberado para institucionalizar los vínculos de los hogares migrantes con circuitos globales de capital y financieros. Estos esfuerzos están fundamentados en el reposicionamiento de la vivienda como bien de consumo a un bien de inversión y motor del crecimiento económico, y del sector financiero como el principal medio de los hogares para acceder a vivienda pública y privada, y a otros servicios básicos. Estas acciones han tenido lugar en el contexto de un proceso más amplio de financialización de la agenda global de pobreza durante las últimas tres décadas (Roy, 2010).

Este artículo se divide en tres partes: la primera revisa la literatura abundante y diversa sobre la economía política de la vivienda en América Latina, producida principalmente por geógrafos en los ańos ochenta y noventa. El objetivo es enmarcar estos nuevos hechos en un contexto histórico-político más amplio. La segunda parte ofrece una breve historia de la política de vivienda y del mercado hipotecario en Colombia. La última parte explora la forma en que el Gobierno colombiano ha tratado de incorporar a los migrantes a una nueva política nacional de vivienda, particularmente a través de la promoción de programas de remesas para el desarrollo.

En términos metodológicos, este artículo se basa en datos empíricos recogidos en ambas puntas de la cadena migratoria entre Colombia y el Reino Unido, por un periodo de seis meses, entre 2008 y $2009^{[2]}$. Consiste en un enfoque de métodos mixtos que incluye entrevistas semiestructuradas en profundidad, observación participante y análisis de datos cualitativos y cuantitativos secundarios. Se realizaron un total de 58 entrevistas a una serie de actores como hogares migrantes, académicos, líderes locales, representantes de organizaciones no gubernamentales, funcionarios del

\footnotetext{
2 La elección de este estudio de caso se debe a la reciente importancia adquirida por los flujos migratorios a este destino: el Reino Unido es el segundo destino más importante de los colombianos en Europa (después de España) y la cuarta fuente de remesas al país. Aunque no se sabe con exactitud cuántos colombianos viven en el Reino Unido, las estimaciones sugieren que alrededor de 70.000 colombianos viven y trabajan en Londres, lugar preferido de los migrantes una vez radicados en este país (Guarnizo, 2008). Sin embargo, un estudio más reciente estimó que el tamaño de la comunidad latinoamericana en Londres es de aproximadamente 113.500 (McIlwaine, Cock y Linneker, 2011).
} 
Gobierno y miembros de entidades privadas del sector de remesas y de la construcción.

\section{LA ECONOMÍA POLÍTICA DE LA VIVIENDA EN AMÉRICA LATINA}

El Banco Mundial y otras IFI han tenido una larga historia de influencia en los programas sociales, económicos y políticos de los países de América Latina. La reorganización sociopolítica que tuvo lugar después de la Segunda Guerra Mundial, vio la consolidación del discurso del desarrollo y la planificación como la solución al problema de la pobreza y la falta de desarrollo en toda la región (Escobar, 1989, 1995). Este discurso del desarrollo ${ }^{3}$ se basaba en la teoría neoclásica del crecimiento económico y consideraba el "subdesarrollo" como un proceso unilateral de acumulación de capital y de progreso técnico que ignoraba las diferencias sociales, políticas y culturales de los países. La adhesión a este paradigma de desarrollo significaba poner énfasis en el ahorro, la inversión de capital y el aumento de la productividad como los principales vehículos para el crecimiento económico y la adopción de una serie de estrategias de desarrollo que fueron concebidas y modeladas en el contexto social y económico, y en la experiencia histórica del norte global (Escobar, 1995). En grande parte, la política de vivienda ha sido diseñada en el marco de la economía política de esta visión del desarrollo, la cual -en general-, no solamente ha ignorado las características particulares del aparato social, económico y político de la región, sino que también ha dejado muy poco espacio para la articulación de enfoques alternativos.

En la época de la posguerra, el ritmo acelerado de la industrialización en las principales ciudades de América Latina provocó un aumento de la demanda de puestos de trabajo en una amplia gama de sectores tales como el manufacturero, comercial y financiero, que trajo consigo un éxodo de las zonas rurales a las urbanas. Sin embargo, problemas estructurales tales como bajos salarios, una distribución desigual de la riqueza, una alta concentración de la tenencia de la tierra y un sector de la construcción que favorecía a los más ricos, significó que esta nueva clase de trabajadores no podía darse el lujo de adquirir vivienda formal (Gilbert, 1998; Gilbert y Ward, 1982). Dado que por estas épocas el foco era la producción industrial, la vivienda no era vista como impulsora del crecimiento sino como un bien de consumo. Aunque esto cambiaría radicalmente cuatro décadas más tarde, por estas épocas, los gobiernos latinoamericanos no fueron incitados por las instituciones financieras internacionales para invertir en el sector (Gilbert y Ward, 1985; Jones y Datta, 1999). En consecuencia, en las principales ciudades de América Latina,

\footnotetext{
3 De acuerdo con Saldańa-Portillo (2003), este discurso surgió como una respuesta a la crisis de expansión del capitalismo de Estados Unidos provocada por el fin de la Segunda Guerra Mundial. Como tal, este discurso del desarrollo surgió como un "discurso complementario" para sustituir la "misión civilizadora” colonial en las economías recientemente emancipadas.
} 
la población más pobre tuvo que recurrir a la construcción de asentamientos "ilegales" para satisfacer sus necesidades de vivienda (Gilbert, 1987; Varley, 1998) ${ }^{4}$.

Entre mediados de los ańos sesenta y la década de los ochenta, los gobiernos de la región mantuvieron un enfoque mixto en la política de vivienda, que consistía principalmente en la provisión de vivienda pública, que no alcanzaba a suplir la enorme demanda, y una postura cambiante motivada por cuestiones políticas en relación con programas de inversión en los asentamientos ilegales (proyectos de mejoramiento mediante la provisión de infraestructura) y proyectos de lotes y servicios (asentamientos donde el Estado entregaba lotes baratos y una gama de servicios básicos, y la construcción quedaba a cargo de los residentes) (Gilbert, 1997; Gough, 1999). A finales de los años ochenta, los efectos negativos de la crisis de la deuda se generalizaron y la tendencia de aumento de las tasas de tenencia de vivienda, principalmente de construcción propia, parecía revertirse debido a una combinación de salarios decrecientes, falta de tierras accesibles para viviendas de bajos ingresos, políticas estatales no tan favorables, creciente comercialización del suelo y un aumento de sus precios (Gilbert y Varley, 1991).

En la década de los noventa, el espantoso estado de las finanzas públicas en la mayoría de los países de la región, resultado de la crisis de la deuda, proporcionó la excusa perfecta para la introducción de políticas de vivienda favorables al mercado coherentes con la nueva ideología de Estado mínimo promovido por el Banco Mundial y las principales agencias de desarrollo. Este proceso había comenzado a principios de 1980, con base en la creencia de que el financiamiento de vivienda, en lugar de la provisión pública de la misma, resolvería la crisis del sector en estos países. La nueva política de vivienda, conocida como el modelo de subsidio de capital para vivienda (Capital Housing Subsidy Model), puso un mayor énfasis en el financiamiento de vivienda y la gestión urbana. Su objetivo era incorporar al sector privado al mercado de la vivienda social, promover la transparencia y ayudar explícitamente a los pobres. En términos generales, la política estaba orientada a reformar la estructura económica y financiera de los países que la adoptaron y a promover el crecimiento económico (Gilbert, 2004; Jones y Datta, 1999; Jones y Ward, 1994a; Jones y Ward, 1994b). Por un lado, este modelo transferiría la provisión de vivienda pública al sector privado, el cual sería "más eficiente" dando "más opciones" a los pobres. Por otro lado, en el marco de este modelo se darían subsidios a los pobres para comprar las viviendas construidas por el sector privado. El Gobierno Pinochet fue pionero en la implementación de este modelo de política de vivienda en 1977 y este fue adoptado (total o parcialmente) por otros países latinoamericanos como Colombia,

\footnotetext{
4 El persistente fracaso de las políticas de vivienda de la región para suplir adecuadamente la gran demanda por viviendas de bajos ingresos ha hecho que esta tendencia histórica continúe hasta hoy, y que iniciativas individuales de construcción de vivienda continúen predominando en los centros urbanos.
} 
Costa Rica, Ecuador ${ }^{5}$ y Panamá como parte del proceso de reformas de ajuste estructural en la década de los noventa (Gilbert, 2004; ONU-Hábitat, 2005; Winchester, 2005).

Este enfoque ahistórico, que establece un modelo único para la formulación de políticas, ha sido una característica distintiva de la agenda mundial para el desarrollo de la posguerra, por lo que las políticas siguen siendo trasplantadas de un lugar a otro sin tener en cuenta las especificidades del entorno social, económico y político de los lugares en los que se implementan (Bondi y Laurie, 2005). Aunque estas políticas ya no se aplican a través de la ejecución de los programas de ajuste estructural, discursos de desarrollo globales siguen siendo difundidos por los organismos multilaterales a través de "modelos de desarrollo y soluciones socio-técnicas preferidas como programas de microcrédito, pactos necesarios para regularizar la propiedad en los asentamientos informales y programas de transferencias monetarias condicionadas" (Peck y Theodore, 2010, p. 171).

En este contexto, se argumenta que la introducción del modelo de subsidio de capital para vivienda representó mucho más que una simple transferencia de responsabilidad del Estado hacia el sector privado. Dos cambios radicales e importantes fueron introducidos al funcionamiento del mercado de la vivienda: el crédito hipotecario se convirtió en la única vía de los pobres para acceder a la vivienda formal y el estatus de la misma pasó de ser un bien de consumo a un vehículo de inversión y un factor clave del empleo y del crecimiento económico. Estos cambios son parte de mudanzas más amplias en las políticas y prácticas del desarrollo que han tenido lugar en las últimas tres décadas, las cuales han tratado de integrar a los pobres a los mercados financieros como la clave para el desarrollo (Roy, 2010). Sin embargo, como se ilustrará con el caso colombiano, el desajuste entre la oferta y la demanda de vivienda y la profunda desigualdad socioeconómica del país ponen en evidencia el hecho de que la lógica del mercado es insuficiente para proporcionar soluciones a las personas que se propone ayudar.

A pesar de que el sector de la vivienda no se convirtió explícitamente en un motor del crecimiento económico hasta la introducción de reformas macroeconómicas de ajuste estructural después de la crisis de la deuda, los gobiernos de América Latina han usado históricamente al sector como una herramienta política ${ }^{6}$. Como Gilbert y Varley (1991, p. 10) han señalado, "el tema de la vivienda difícilmente puede estar divorciado del ámbito político en América Latina, donde el Estado ha manipulado las poblaciones de bajos ingresos con fines políticos”. Además, los gobiernos de toda la región se han dado cuenta de los beneficios que el aumento de

\footnotetext{
5 Para una evaluación completa del programa Ecuatoriano "Sistema de incentivos para vivienda”, ver Klaufus (2010a).

6 Gilbert y Varley (1991) y Varley (1998) proporcionan una excelente explicación de los usos políticos de la política de vivienda en México.
} 
las tasas de tenencia de vivienda podrían traer en términos de estabilidad social y política $y$, por tanto, han fomentado activamente las bondades de tener casa propia ${ }^{7}$. No debe sorprender entonces que varios gobiernos hayan intervenido activamente para favorecer a este sector, especialmente en tiempos de recesión económica, y que el uso populista de la política de vivienda suela ser una de las instancias en las que los gobiernos aparenten intervenir en nombre de los pobres (Gilbert y Ward, 1985).

Otro aspecto importante que debe ser considerado en este uso político de la política de vivienda es el deseo expresado por la mayoría de personas de tener casa propia. Estudios previos han encontrado que la mayoría de la gente en las ciudades latinoamericanas desea/ sueña con tener casa propia (Gilbert y Varley, 1991). Según Gilbert (1987, p. 47), este deseo va más allá de meras aspiraciones y "es en parte un reconocimiento de la realidad económica; en parte, una consecuencia del deseo de ser independiente... pero también es una actitud estimulada por la ideología del capitalismo, reforzada continuamente por la publicidad televisiva y las telenovelas". Por tanto, es razonable argumentar que la promoción de los gobiernos de las bondades de tener casa propia como parte integral de las políticas nacionales de desarrollo influye, y es a la vez influenciada, por el deseo de la gente de tener vivienda propia y es difícil saber dónde uno comienza a imponerse sobre el otro.
Aunque la política estatal de vivienda es un determinante crítico de su tenencia, hay muchos otros factores que influyen en ella. Varios estudios sobre el mercado de vivienda urbana en América Latina han llegado a la conclusión de que la decisión de los hogares de comprar o alquilar una casa también está determinada por las diferentes opciones y limitaciones que enfrentan dado su contexto socioeconómico. Los precios del suelo, el costo de los materiales de construcción, los ingresos, los costos y la disponibilidad de transporte y el trade-off entre alquilar y comprar parecen ser ingredientes importantes en esta mezcla (Gilbert y Varley, 1991; Gilbert y Ward, 1985). Pero como Smith (2008) y Smith, Munro y Christie (2006) han señalado, los mercados son socialmente construidos y el de vivienda tiene un elemento no monetario explícito que lo hace profundamente arraigado en una red de relaciones sociales; por tanto, su dinámica solo puede entenderse a la luz de la economía política más amplia.

Esta breve reseña histórica de la política de vivienda en América Latina ilustra la importancia vital que los factores económicos y no económicos han jugado en la definición de las políticas públicas en la región. Así, la política de vivienda no está determinada únicamente por la situación del mercado de vivienda de un país, sino que está fuertemente influenciada por otros factores como la ideología sociopolítica de los gobiernos, el grado de influencia

\footnotetext{
El fomento de estas ideas no es exclusivo de los gobiernos latinoamericanos. Muchos otros gobiernos de países en desarrollo y desarrollados han hecho de la promoción de la vivienda propia un tema central de sus políticas de desarrollo nacionales. Para un panorama global, véase Gilbert (2008); para el caso de Estados Unidos, véase Smith (1988); para el caso del Reino Unido, véase Smith (2008) y Smith, Easterlow y Munro (2004).
} 
ejercida por los organismos internacionales en las políticas públicas, la capacidad de los grupos de interés para influir en ellas y la centralidad otorgada al sector de la vivienda para la estabilidad social y política de un país.

Por tanto, la transformación de la vivienda en un elemento de inversión y motor del crecimiento económico y del desarrollo ofrece un claro ejemplo de las recientes transformaciones en la economía política del Estado y pone de manifiesto una de las formas en que se está movilizando el poder del Estado para la expansión de algunos intereses y la consolidación de un modelo específico de desarrollo. Sin duda, estos esfuerzos han sido apoyados por la reproducción de un discurso mediático que iguala el poseer casa propia con atributos tales como responsabilidad, independencia y ejercicio de la ciudadanía.

\section{LA POLÍTICA DE VIVIENDA Y EL MERCADO HIPOTECARIO EN COLOMBIA: BREVE HISTORIA}

En los últimos cincuenta años, Colombia se ha transformado de una sociedad rural a una urbana: el $75 \%$ de la población del país vive en zonas urbanas y se espera que el $81 \%$ lo hará en 2030 (onU-Hábitat, 2012). Esta rápida transición ha puesto una gran presión sobre la demanda y ha generado un agudo déficit cualitativo (calidad de las viviendas existentes) y cuantitativo (número de viviendas existentes). A pesar de que en virtud de la Constitución colombiana de 1991, "todos los ciudadanos colombianos tienen derecho a una vivienda digna” y el Estado se compromete a "establecer las condiciones necesarias para que este derecho se torne una realidad"8, el déficit nacional de vivienda ha aumentado sustancialmente en los últimos años. Se estima que se necesitan alrededor de tres millones de viviendas - cerca de un tercio del total de hogares del país- para alojar a cada familia. El déficit de vivienda de interés social (vis) es de alrededor de 1,2 millones de unidades y el 1,9 millones restantes representa el déficit para todos los otros tipos de vivienda (Clavijo, Janna y Muńoz, 2005; El Espectador, 2010b).

Hasta 1990, el enfoque de la política de vivienda de los gobiernos nacionales siguió el de la mayoría de los países de América Latina: el mercado de vivienda estaba caracterizado por un sector de construcción formal que suplía las necesidades de la clase media y una agencia estatal de vivienda pública que construía un número insuficiente de casas para los pobres, lo que produjo la proliferación de iniciativas individuales de construcción de vivienda por parte de los hogares de bajos ingresos.

Durante el Gobierno de César Gaviria (1990-1994), la política de vivienda de Colombia cambió radicalmente. El Plan Nacional de Desarrollo de Gaviria, "La revolución pacífica", estableció un enfoque de desarrollo que se tradujo en una serie de políticas económicas neoliberales (la apertura económica) que buscaban integrar a Colombia al sistema capitalista mundial. Esto fue de hecho una revolución que le dio carta blanca al Gobierno para liberalizar

8 Artículo 51, Constitución Política de la República de Colombia (1991). 
el comercio, eliminar todas las restricciones a la inversión extranjera directa, reformar el código laboral y el sistema de seguridad social y privatizar la salud, la educación, el transporte y otros servicios básicos (Gilbert, 2004).

Bajo este escenario, el Gobierno adoptó rápidamente el modelo de subsidio de capital para vivienda impulsado por el Banco Mundial y las principales agencias de desarrollo que buscaba "alentar al Gobierno a desempeñar un papel facilitador: dejando de lado la construcción, financiación y mantenimiento de la vivienda, y enfocándose en el mejoramiento de la eficiencia del mercado de vivienda y las condiciones de vivienda de los pobres" (Banco Mundial, 1992, citado en Gilbert, 1998, p. 100 ). Bajo este modelo, el Gobierno colombiano dejó de construir y financiar viviendas públicas y lo más importante, permitió al sector privado participar plenamente en la construcción y financiamiento de vivienda de interés social (vis). El nuevo papel del Gobierno era el de asignar subsidios (subsidio familiar de vivienda - SFv) a los hogares de bajos ingresos para la adquisición de viviendas construidas por el sector privado, por lo que el Estado se convirtió efectivamente en un mediador entre la industria privada y los hogares. Para tener derecho a un subsidio, las familias tenían que cumplir con un estricto conjunto de requisitos tales como tener una cierta cantidad de ahorros y estar dispuestos a sacar una hipoteca, entre otros?
Sin embargo, la demanda del subsidio familiar de vivienda ha sido persistentemente mayor que el número de subsidios asignados. A modo de ejemplo, se estima que el $80 \%$ de los hogares urbanos que no son propietarios de vivienda pertenecen a los estratos bajos y medio-bajo (1, 2 y 3) y, por tanto, podrían calificar para un subsidio; pero, en promedio, menos del $10 \%$ de los hogares que solicitan un subsidio de hecho lo reciben (Conpes, 2002). Además, las deficiencias del SFV van más allá del desequilibrio entre la oferta y la demanda de subsidios. Dado que el modelo requiere que las familias saquen una hipoteca para financiar la diferencia entre el precio de mercado del inmueble y el subsidio, muchas familias pobres a las que se les otorga el subsidio no pueden utilizarlo. Existen tres razones principales para explicar esta situación: las familias no tienen suficientes fondos para cubrir la cuota inicial de la casa (por lo general, el 30\% del valor del inmueble), sus bajos ingresos no les permiten efectuar los pagos mensuales de la hipoteca o incluso si cumplen con todos los requisitos, hay una escasez de viviendas asequibles. Esto se debe a que las empresas constructoras privadas tienen poco o ningún incentivo para construir vIs dado que este tipo de vivienda tiene muy poco valor agregado y no ofrece mucho en términos de ganancias. Así, el programa de subsidios obliga a los pobres a depender del sector financiero para acceder a una vivienda social, pero dada la realidad socioeconómica

\footnotetext{
9 La asignación de los subsidios se da a través de la evaluación de los puntajes de los hogares solicitantes. Estos se basan en aspectos tales como el ingreso, si el solicitante es una mujer cabeza de familia, y la cantidad y antigüedad de los ahorros depositados en cuentas de ahorro programadas. El peso que se da a esta última variable se ha incrementado considerablemente desde 2001.
} 
del país, la mayoría de estos hogares apenas gana suficiente dinero para cubrir sus necesidades básicas y, en general, no tiene la capacidad de acumular suficientes ahorros para convertirse en propietarios de vivienda.

Aunque las administraciones posteriores han hecho algunos cambios al modelo de subsidios, los elementos clave del modelo implementado en la década de los noventa permanecen intactos. Más recientemente, el Gobierno de Álvaro Uribe (2002-2010) reconoció explícitamente en su Plan Nacional de Desarrollo a los sectores de la vivienda y de la construcción (y, por extensión, al sector financiero) como importantes impulsores del crecimiento económico. Aunque el Gobierno de Uribe se comprometió, entre otras cosas, a aumentar el financiamiento de vivienda de interés social y promover una ley que institucionalizara las microfinanzas para la vivienda (CONPES, 2002), el gasto público social en vivienda se mantiene entre los más bajos de América Latina, un promedio de 0,6\% del pIB para el periodo 2002-2010 ${ }^{10}$ (CENAC, 2011a; CePAl, 2012). En contraste, el hecho de dar mayor énfasis a las variables que miden el "esfuerzo personal" de los hogares en lugar de medidas más estructurales como la marginalización socioeconómica de los hogares pobres para la asignación de los subsidios, la continuación de esta política de vivienda es una indicación más de que el Gobierno promueve el aumento de las tasas de tenencia de vivienda como parte de una agenda más amplia que equipara tener casa propia con atributos como la responsabilidad, la independencia y el ejercicio de la ciudadanía.

En cuanto al mercado hipotecario de vivienda, no mucho ha cambiado desde la década de los noventa: bancos privados nacionales y transnacionales suministran la mayor parte de las hipotecas; solo hasta el $70 \%$ del valor de mercado del inmueble puede ser financiado (80\% en el caso de vis); el periodo de amortización oscila entre 5 y 30 años, y las tasas de interés reales se mantienen altas, entre 9 y $13 \%$ anual (Gómez, Bougher y Robertson, 2005).

En 2009, el Gobierno de Uribe introdujo un nuevo conjunto de incentivos para las personas que desearan invertir en una casa nueva, al subsidiar las tasas de interés de los préstamos de vivienda, independientemente de los ingresos de la persona. Los subsidios se administraban y se asignaban a través de la banca privada y el único requisito era que la persona interesada informara al banco al momento de firmar el contrato de hipoteca. El Gobierno luego asumía una parte de la deuda en cuestión (3 a 5\% de la tasa de interés) durante los primeros siete años de la hipoteca (CENAC, 2011b; MIJ, 2009) ${ }^{11}$. En los primeros tres meses de la introducción de este esquema, se habían asignado 15.000 de los 32.000 subsidios disponibles. Este tipo de política de subsidio de hipotecas sin

\footnotetext{
10 En la región, solo Chile, Paraguay, Ecuador y Honduras gastan menos en vivienda social.

11 La tasa de interés promedio para crédito hipotecario nuevo se situó en 9,7\% en 2009. En cambio, la tasa correspondiente a la Unión Europea fue del 2,7\%.
} 
condiciones pone en evidencia que la política de vivienda sigue estando sesgada hacia las clases media y media-alta, las cuales están en una posición privilegiada para acceder al financiamiento de vivienda formal, pero solo constituyen un porcentaje muy pequeño de la población residente en el país y en el exterior.

La insistencia del Gobierno en subvencionar solamente vivienda nueva financiada con hipoteca y la ausencia de focalización hacia grupos de bajos ingresos, hacen evidente que estas medidas agresivas tienen como principal propósito promover la construcción de viviendas con más valor agregado, creando así oportunidades de alta rentabilidad para el sector de la construcción y el financiero. Este hecho es corroborado abiertamente por empresarios del sector de vivienda, que admiten que esta nueva modalidad de subsidio no solo permite mayores ganancias, sino que también envía un mensaje claro sobre el compromiso del Gobierno para establecer las condiciones necesarias para que el sector prospere ${ }^{12}$.

El primer mandato del actual Gobierno de Juan Manuel Santos (2010-2014) no solo extendió la política de subsidio de hipotecas sin condiciones, sino que también ubicó a la vivienda como uno de los principales pilares de su Plan Nacional de Desarrollo "Prosperidad para todos". El Gobierno se comprometió a incrementar el inventario nacional en un millón de nuevas viviendas "dando a los colombianos las herramientas para adquirirlas". Los nuevos mecanismos para facilitar la adquisición de vivienda iban desde la creación de subsidios adicionales para la vivienda de interés social y el estímulo a la construcción de viviendas para alquiler hasta proporcionar incentivos para que los bancos le prestaran a los pobres (Dinero, 2011a, 2011b). Esta estrategia en particular-la incorporación de los pobres al sector financiero-, parece ser uno de los principales objetivos económicos del Gobierno Santos. Pocos días después de que asumió el cargo, Santos instó al sector financiero a esforzarse a diseñar los instrumentos necesarios para "bancarizar" a los colombianos que aún no son parte del sistema. Haciendo paralelos con la operación militar que liberó a 15 rehenes en poder de la guerrilla cuando era ministro de Defensa en 2008, Santos declaró: "tenemos que pensar lo impensable"; "este mandato de cuatro años será de bancarización y de profundización de los servicios financieros" (El Espectador, 2010a). Como parte de estos objetivos, el Plan Nacional de Desarrollo de Santos también incluyó una sección entera sobre "educación financiera" para las familias migrantes, cuyo objetivo era "facilitar y ampliar el acceso a servicios financieros como el ahorro, la inversión, el crédito y los seguros a los receptores y remitentes de remesas" y "facilitar la inclusión financiera de los emigrantes en su país de residencia" (PND, 2010).

Sin embargo, a pesar de todos estos esfuerzos, la cartera hipotecaria de vivienda en el país no ha aumentado: el crédito hipote-

12 Entrevista con el dueño de una empresa de construcción importante en la zona cafetera y miembro de la Cámara Colombiana de la Construcción (CAmacol). Eje Cafetero, 6 de julio de 2009. 
cario sigue representando solo una modesta proporción del PIB, en torno del $4 \%$, y $12,6 \%$ del crédito total, y la tasa de tenencia de vivienda se redujo de alrededor de $66 \%$ en la década de los ochenta, a 50\% en 2007 (CENAC, 2011 a; Clavijo et al., 2005) ${ }^{13}$. Esto parece ser el resultado de otros problemas estructurales como la baja demanda de crédito asociado a bajos niveles de empleo formal y actividad económica, y la falta de voluntad por parte de los intermediarios financieros de extender crédito a amplios sectores de la población (CONPES, 2002).

En resumen, a pesar de los diversos esfuerzos para posicionar al sector de la vivienda como un pilar del crecimiento económico, el mercado hipotecario de vivienda en Colombia continúa siendo superficial. La rigidez de los requisitos y los bajos niveles de empleo formal y de actividad económica han hecho que en la práctica, el financiamiento de vivienda siga estando fuera del alcance de la mayoría de la población colombiana, que pertenece a los estratos de ingresos bajos y medio-bajo. Así, las preguntas que surgen son ¿cómo encajan los migrantes en esta dinámica? ¿Son los intentos agresivos del Gobierno para promover el mercado de vivienda a nivel internacional, una respuesta al éxito limitado de estos esfuerzos en los últimos treinta años?

\section{LOS MIGRANTES COMO SUJETOS DE UNA POLÍTICA DE VIVIENDA NUEVA Y REFORMADA}

Dado el reconocimiento de los migrantes como una fuerza económica y política, muchos gobiernos latinoamericanos están intentando sacar provecho de las conexiones de los hogares migrantes con circuitos globales de capital y financieros, tratando de institucionalizar los flujos de remesas ${ }^{14}$ y las actividades socioeconómicas transnacionales que se derivan de ella, especialmente aquellas consideradas como "inversiones productivas". Si bien el Banco Mundial ha estado a cargo de coordinar estos esfuerzos, una variedad de políticas y programas de migración para el desarrollo está siendo promovida a escala global por una gama de organizaciones internacionales y de think tanks, a las cuales se les ha dado relevancia internacional a través del Diálogo de Alto Nivel sobre la Migración Internacional y el Desarrollo de las Naciones Unidas y la creación del Grupo Mundial sobre Migración, al interior de la onu (Gamlen, 2014). Como lo ilustra el caso colombiano, estas políticas y programas reproducen un discurso que traslada la responsabilidad del desarrollo del Estado a sus ciudadanos residentes en el país y en el exterior, insiste en la primacía del sector financiero para el acceso de los hogares

\footnotetext{
13 Las tasas para Chile, que fue pionera del modelo de subsidio de vivienda, son, respectivamente, $19 \%$ del PIB, $25 \%$ de la cartera total y cerca del $70 \%$ de tenencia de vivienda.

14 Las remesas hacia América Latina y el Caribe representan alrededor del $70 \%$ de los flujos de inversión extranjera directa (IED) y exceden los flujos de asistencia oficial para el desarrollo (AOD) en un $500 \%$ (World Bank, 2006, 2011).
} 
a servicios básicos y promueve la inversión "productiva” (en vivienda) como el mecanismo por excelencia para hacer uso "apropiado" de las remesas. El nuevo entusiasmo en torno a este tipo de programas de migración para el desarrollo está fundamentado en intereses políticos y económicos, y exagera los beneficios y los efectos multiplicadores que las remesas pueden tener en los países de origen de los migrantes (De Haas, 2012; Gamlen, 2014). Sin embargo, el discurso de migración para el desarrollo y sus políticas se ajusta a los intentos de los gobiernos del Norte para controlar y manejar la migración internacional (Datta, 2009; Delgado-Wise y Márquez Covarrubias, 2010; Gamlen y Marsh, 2011) y a una ideología que busca "amplificar el neoliberalismo", es decir, busca la creación de nuevas formas globales de gestión económica tecnocrática y de políticas sociales invasivas (Gamlen, 2010; Peck y Tickell, 2002, pp. 388-389).

En este contexto, el Gobierno colombiano ha tratado de sacar provecho de los vínculos transnacionales de los migrantes al intentar hacerlos parte integral de una definición renovada de la nación. Para este efecto, el Gobierno ha cambiado radicalmente sus políticas hacia sus ciudadanos residentes en el exterior. Aunque los colombianos adquirieron el derecho a la doble nacionalidad en la década de los sesenta, desde la implementación de la nueva Constitución en 1991, los colombianos residentes en el extranjero han adquirido los derechos de voto en el exterior, representación en el Congreso Nacional y el derecho a ser elegidos para el Congreso (Guarnizo, Sánchez y Roach, 1999).

En los últimos quince años, el Gobierno colombiano ha promovido la agenda de migración para el desarrollo y ha intentado redefinir su relación con la diáspora mediante el diseño de programas para canalizar las remesas a sectores clave como el de vivienda y financiero, principalmente a través del programa "Colombia nos une". Este programa tiene como objetivo "vincular permanentemente al Estado colombiano con sus ciudadanos residentes en el exterior", "reconocerlos como una parte vital de la nación" y "garantizar los mecanismos para facilitar el envío de remesas y para canalizarlos hacia el ahorro y la inversión", entre otros (MRE, 2004).

El Gobierno ha promovido la canalización de las remesas hacia la inversión "productiva” principalmente a través de programas de remesas para vivienda, tales como " $\mathrm{Mi}$ casa con remesas" y las ferias internacionales de vivienda. "Mi casa con remesas" es un programa diseńado y financiado por el Banco Interamericano de Desarrollo (BID) por medio del cual los hogares receptores de remesas pueden solicitar un crédito hipotecario para comprar viviendas populares nuevas. En Colombia, el programa fue implementado a través de una alianza entre el BID, las Cajas de Compensación Familiar y Bancolombia. El programa surgió como parte del clúster de proyectos del BID que buscaba atraer los flujos de remesas hacia canales formales con el fin de fortalecer el sistema financiero global ${ }^{15}$. Pero el

15 El BID financió cinco proyectos de remesas para vivienda en América Latina: uno en Colombia, dos en México, 
Gobierno colombiano también fue el primero en América Latina en patrocinar, logística o financieramente, ferias de vivienda para los colombianos en el exterior ${ }^{16}$. Estas ferias se han llevado a cabo desde 2005 en las principales ciudades de destino de los colombianos en el Norte: Nueva York, Miami, Madrid y Londres. El propósito de estas ferias es permitirle al sector de la construcción llevar su portafolio inmobiliario directamente a los colombianos en el exterior, usando el respaldo del Gobierno para generar confianza entre los migrantes.

Las ferias son organizadas por la Cámara Colombiana de la Construcción (CAMACOL) o por la Lonja, una organización que agrupa a constructores y agentes inmobiliarios y requieren un esfuerzo logístico monumental. El apoyo del Gobierno se ha hecho a través de los consulados, las embajadas y Proexport, la agencia gubernamental para la promoción de las exportaciones, el turismo y la inversión extranjera. Los cónsules y embajadores suelen asistir a las ferias y la de Nueva York en el año 2005 contó con la presencia del presidente de la república. Estas ferias son ampliamen- te difundidas en una variedad de medios de comunicación local para "quienes están lejos del hogar y quieren invertir en su patria” ${ }^{17} \mathrm{y}$, por lo general, los panfletos publicitaros hacen uso intensivo de la bandera nacional.

El argumento en pro de estas ferias es coherente con la idea que prevalece entre funcionarios gubernamentales y las personas involucradas en el negocio de las remesas, de que estas tienen que ser canalizadas hacia el "uso productivo" dado que están siendo "desperdiciadas en consumo". Así lo señala de manera elocuente el dueño de una empresa de construcción en la zona cafetera, quien ha participado en las ferias desde que comenzaron:

El fenómeno que ha ocurrido en los últimos años y sigue ocurriendo hoy, es que la gente envía dinero a sus familiares y el dinero se pierde... y este es uno de los fenómenos persistentes con relación a las remesas... así que lo que tratamos de hacer a través de las ferias es canalizar toda esa inversión por parte del migrante para que pueda ser aplicada a las sinergias de la economía regional y que no se gaste solo en bares y ese tipo de $\operatorname{cosas}^{18}$.

uno en El Salvador y uno en Ecuador. Además, desde el 2009, el banco ha financiado numerosos estudios, encuestas y conferencias, y 45 proyectos nacionales y regionales de asistencia técnica para promover las remesas como una herramienta para el desarrollo (Hall, 2010).

16 Utilizando el mismo tipo de discurso, este tipo de ferias se está convirtiendo en el instrumento favorito de muchos gobiernos de América Latina: México patrocinó la primera feria de vivienda, "Tu vivienda en México", en Estados Unidos en el 2007 y Honduras lo hizo en el 2011. Del mismo modo, Ecuador promovió la primera feria en Europa (Madrid y Barcelona), "Mi casa en Ecuador", en 2006 y en Estados Unidos en el 2008 y, en 2006, El Salvador comenzó a organizar una serie de ferias en Estados Unidos (Klaufus, 2010b).

17 Este fue el lema de la feria "Dónde vivir e invertir en Colombia", que tuvo lugar en Londres en el 2008.

18 Entrevista, Eje Cafetero, 6 de julio de 2009. Esta idea generalizada sobre las remesas ha sido articulada explícitamente, no solo en numerosos documentos oficiales, sino que también fue reiterada por algunos actores claves y otros ciudadanos con los cuales interactué durante mi investigación en Colombia. 
Durante las ferias inmobiliarias se canta el himno nacional y se realizan una serie de presentaciones donde se destaca cómo los migrantes pueden contribuir a la economía colombiana haciendo uso de canales formales para el financiamiento de vivienda, mientras se alude a su lealtad hacia sus familias y a un futuro retorno a una Colombia "nueva" y más segura. A pesar de la crisis económica mundial de 2008, las ferias continuaron realizándose. Por ejemplo, CAMAcol organizó ferias anualmente entre el 2009 y el 2012 en el área triestatal de Nueva York-Nueva JerseyConnecticut, y en Miami y Houston en 2011. Del mismo modo, la Lonja organizó ferias en Madrid y Londres en el 2010. No obstante, ninguna de las dos asociaciones ha realizado más ferias en Londres desde entonces.

Sin embargo, a pesar de toda la retórica, los migrantes no han sido plenamente reconocidos como una parte vital de la nación y las políticas públicas dirigidas hacia ellos han sido selectivas y han privilegiado no la promoción de los derechos sociales de los migrantes sino su papel como consumidores e inversionistas. A nivel nacional, está claro que hay algunos requisitos que limitan el acceso de los migrantes al programa de subsidio familiar de vivienda actual. En particular, uno de los requisitos para solicitar el SFV es que los miembros de la familia tienen que compartir el mismo espacio habitacional y si se le otor- ga el subsidio, el beneficiario debe ocupar la propiedad y no puede venderla en los cinco años siguientes a la concesión del beneficio. Dada su dispersión geográfica y el carácter semipermanente de los flujos migratorios colombianos, los colombianos que residen en el exterior no tienen la oportunidad real de acceder al programa de subsidio de vivienda, aun si tienen los recursos para cumplir con los requisitos necesarios para obtener una hipoteca ${ }^{19}$. Por tanto, en la práctica, hay una divergencia entre el discurso del Estado de incluir a los migrantes como una parte integral de la nación y, en vista del creciente transnacionalismo de la sociedad colombiana (Guarnizo, 2006), su limitada definición de lo que constituye un hogar.

A nivel internacional, los migrantes que adquieren una propiedad en el exterior tienen la opción de registrar la compra como una inversión extranjera, tanto en los casos en que el crédito hipotecario es conseguido a través de una de las instituciones financieras en Colombia o en el extranjero. El registro de la transacción como una inversión extranjera garantiza al comprador condiciones favorables para la eventual repatriación de los beneficios obtenidos de la propiedad ${ }^{20}$. Debido a que este registro implica gastos adicionales para el comprador, la mayoría de los colombianos en el exterior no registran sus compras de propiedad como tal. Así, aunque

\footnotetext{
19 Por el contrario, el Gobierno de la República Dominicana estableció un programa para los migrantes que deseen regresar a la isla y cumplan con algunas condiciones, dándoles preferencia en el acceso a viviendas construidas para este propósito y otorgándoles el $60 \%$ de la cuota inicial (Levitt, 2001b).

20 Estos beneficios incluyen la exención de impuestos de entrada y salida del capital del país.
} 
no hay limitaciones legales que impidan a los colombianos adquirir vivienda desde el exterior, en la práctica es un proceso bastante difícil y desalentador. Por un lado, tienen que incurrir en altos costos adicionales, tales como nombrar un representante legal en Colombia para llevar a cabo los procedimientos administrativos y legales de la transacción, pagar los costos del envío de los pagos de la hipoteca, sobrecostos de evaluación de crédito y servicios postales, entre otros ${ }^{21}$. Por otro lado, uno de los mayores obstáculos para los migrantes que deseen comprar una casa en Colombia es su incapacidad para obtener financiamiento de vivienda, en el país o en el extranjero, ya sea porque carecen de estatus legal en el país de residencia ${ }^{22}$ o no pueden demostrar que tienen la capacidad de hacer esa inversión de largo plazo. Esto se explica parcialmente por el hecho de que actualmente para obtener un crédito hipotecario, los bancos nacionales y extranjeros requieren que el migrante proporcione pruebas de su estatus legal en el país de residencia, certificados de empleo e ingresos, declaraciones de impuestos y extractos de cuentas bancarias, entre otros. Este es uno de los principales problemas citados para explicar el éxito limitado que han tenido los programas de remesas para vivienda. Uno de los representantes del programa "Mi casa con remesas" resumió la situación de esta manera:
Un problema común para nosotros es que la gente tiene el dinero para hacer el pago mensual de la hipoteca, pero no tienen el $30 \%$ para la cuota inicial y quieren pedir prestado el $100 \%$ del precio del inmueble y nosotros no podemos ofrecerles eso debido al alto riesgo que conlleva. Vimos [en la última feria] que hay mucha expectativa: los migrantes quieren comprar una casa porque es su deseo, pues quieren tener su patrimonio en Colombia, ya sea para sí mismos o para sus familias, pero en este momento hay una gran cantidad de inestabilidad y tienen miedo de hacerlo ahora ${ }^{23}$.

Sin embargo, los migrantes parecen estar encontrando mecanismos alternativos para realizar su sueńo de tener casa propia y la evidencia sugiere que el uso de remesas para financiar la inversión en vivienda es significativo. Un estudio de 2004 encontró que cerca del $10 \%$ de los hogares receptores de remesas en la zona cafetera del país utilizan una parte para financiar la adquisición de la vivienda, y el $40 \%$ de ellos las utiliza para hacer ampliaciones o mejoras a sus viviendas (Garay y Rodríguez, 2005). Asimismo, un estudio realizado en 2005 entre los migrantes colombianos que envían remesas desde Estados Unidos encontró que el $74 \%$ de ellos estaba ahorrando dinero para invertir en una casa en Colombia, aunque solo el $24 \%$ tenía confianza en el proceso para realizar

21 Por ejemplo, los costos adicionales de la compra y el financiamiento de una casa nueva para un migrante colombiano en Espańa se han estimado en cerca de 300 \% por encima de los gastos en que incurriría un colombiano residente en el país (Jaramillo y Mejía, 2008).

22 A pesar de que no se tienen datos concretos, se estima que entre el 50 y el $66 \%$ de los colombianos en el exterior no cumplen con las leyes migratorias del país en que residen (Jaramillo y Mejía, 2008).

23 Entrevista, Eje Cafetero, 3 de julio 2009. 
la compra (Gomez et al., 2005). A pesar de la importancia que las remesas han ganado en Colombia en los últimos ańos, llegando a us\$4.093 millones en 2014 (Banco de la República, 2015), no hay datos precisos sobre su impacto en los sectores de la construcción y financiero de Colombia. Solo dos estudios han intentado medir su importancia en el sector de la vivienda: mientras Clavijo et al. (2004) apuntan a que el flujo de remesas es una de las razones para explicar el dinamismo del sector después de la crisis hipotecaria de finales de los años noventa; Cárdenas, Cadena y Quintero (2004) encontraron que las remesas tenían una correlación positiva con la demanda de vivienda a nivel nacional.

Según cifras oficiales, las compras de vivienda realizadas por los colombianos en el exterior sumaron alrededor de us\$1.200 millones entre 2003, año en que comenzaron a registrarse, y 2013 (Collazos, 2015). Sin embargo, la cifra oficial de la cantidad de remesas enviadas para financiar la compra de vivienda es, en promedio, menos de $4 \%$ de la cantidad total de dinero que los migrantes envían anualmente a sus familias en Colombia $^{24}$. El tamaño insignificante de esta cifra no es de extrañar dados los diferentes obstáculos que los migrantes enfrentan en la adquisición y el financiamiento de vivienda y el hecho de que las estadísticas oficiales solo registran las compras de vivienda realizadas a través de los canales formales.

\section{OBSERVACIONES FINALES}

Históricamente, el sector de la vivienda ha tenido una gran importancia política y económica para los gobiernos de América Latina, aunque esto no siempre se ha expresado de forma explícita. El reposicionamiento de la vivienda de un bien de consumo a un bien de inversión y motor del crecimiento económico, así como del sector financiero como el principal mecanismo de los hogares para acceder a la vivienda pública y privada, es solo una de las más recientes manifestaciones de esta realidad. A pesar de los numerosos esfuerzos por parte de sucesivos gobiernos colombianos para posicionar a los sectores de la construcción y financiero como motores de empleo y crecimiento económico en los últimos cuarenta años, la tasa de tenencia de vivienda en Colombia no se ha recuperado y el mercado hipotecario continúa siendo superficial.

En este contexto, el Gobierno colombiano ha adoptado una serie de programas de migración para el desarrollo que buscan canalizar las remesas para sectores específicos como el de vivienda y financiero. Aunque no hay evidencia empírica para sustentar el vínculo entre la migración internacional y el desarrollo económico, estos programas se han convertido en una especie de ortodoxia política internacional, un proceso en el que, si las políticas son "correctas", todos los involucrados se beneficiarán (Delgado-Wise, Márquez Covarrubias y Puentes, 2013; Gam-

24 Fuente: Banco de la República (2015); Collazos (2015). 
len, 2014). Esta nueva ortodoxia se basa en una concepción errónea y unidimensional del desarrollo como una cuestión de "intervención propositiva” (Bastia, 2013; Cowen y Shenton, 1996), y promueve la consolidación de un determinado modelo de desarrollo tecnocrático. Además, tiende a ignorar la naturaleza multidimensional (económica, social, política, geográfica, etc.) de la migración y el desarrollo, y su relación dialéctica con los procesos político-económicos del desarrollo capitalista global (Portes, 2009). Así, la elección de los sectores financiero y de la vivienda para la incorporación de los migrantes a la agenda política nacional no es una coincidencia, sino más bien, un esfuerzo deliberado para institucionalizar las prácticas transnacionales de los hogares migrantes y sus vínculos con circuitos globales de capital y financieros. Como Zapata (2013) ha sugerido, a la luz de la creciente importancia de las remesas como fuente de financiación para los países emisores de migrantes, la nueva ola de entusiasmo en torno al nexo entre migración internacional y desarrollo parece estar impulsada por la necesidad del gran capital financiero de garantizar su reproducción, con la ayuda del Estado, mediante la incorporación de los migrantes al funcionamiento del sistema financiero mundial a través de su representación como "nuevos sujetos financieros transnacionales".

En términos más generales, existe una divergencia entre la retórica del Gobierno de hacer que los migrantes se conviertan en parte integral de la nación colombiana y las medidas adoptadas para hacer esto una realidad, sobre todo en términos de la política de vivienda. Es evidente que hay algunos requisitos que limitan el acceso de los migrantes al programa de subsidio familiar de vivienda actual, programa bandera de la política nacional de vivienda. En particular, los estrictos requisitos y la estrecha definición de lo que constituye una familia, sobre todo en vista del creciente transnacionalismo de la sociedad colombiana, impide que la mayoría de los hogares migrantes participen de él.

Además, a pesar de que el Gobierno colombiano ha tratado insistentemente de promover la idea de que los migrantes pueden actuar como agentes transnacionales del desarrollo y ha puesto todo su peso detrás de la creación y el mantenimiento de un nuevo espacio socioeconómico transnacional para los sectores de vivienda y financiero, especialmente por medio de las ferias de vivienda, el impacto global de estos programas de remesas para vivienda ha sido modesto ${ }^{25}$. Dado el carácter "occidentalizado" de estos programas de desarrollo y la marginal posición socioeconómica que la mayoría de los migrantes ocupa en las sociedades del Norte donde residen, estos esfuerzos se han quedado cortos para satisfacer las necesidades de vivienda de los migrantes y, por eso, no han logrado captar una parte importante de los flujos de remesas.

25 Asimismo, la evidencia reciente indica que, debido a la desconfianza en las instituciones financieras y las altas tasas bancarias, el impacto de las remesas en el desarrollo del mercado financiero de América Latina es menor que en el resto del mundo en desarrollo (Fajnzylber y López, 2007). 
Aunque la compra de vivienda con remesas por parte de los colombianos residentes en el exterior ha mostrado un balance robusto en los últimos años, el monto de las remesas que se registra oficialmente para financiar la compra de vivienda aún es un pequeño porcentaje de la cantidad total de dinero que los migrantes envían a sus familias en Colombia.

\section{REFERENCIAS}

Banco de la República (2015). Ingresos de remesas de trabajadores en Colombia. Recuperado de http:// www.banrep.gov.co/es/remesas

Bastia, T. (2013). The Migration-Development Nexus: Current Challenges and Future Research Agenda. Geography Compass, 7 (7), 464-477.

Bondi, L. y Laurie, N. (2005). Working the Spaces of Neoliberalism: Activism, Professionalisation and Incorporation: Introduction. Antipode, 37 (3), 393-401.

Cárdenas, M., Cadena, X. y Quintero, J. F. (2004). Determinantes de la actividad edificadora en Colombia. Bogotá: Fedesarrollo.

CENAC (2011a). Contexto sectorial internacional: Colombia y América Latina. Bogotá: Centro de Estudios de la Construcción y el Desarrollo Urbano y Regional.

CENAC (2011b). Contexto sectorial internacional: Colombia y Unión Europea. Bogotá: Centro de Estudios de la Construcción y el Desarrollo Urbano y Regional.

cepal (2012). Gasto Social en América Latina y el Caribe (Estadisticas). Washington: CEPAL.

Clavijo, S., Janna, M. y Muñoz, S. (2005). The Housing Market in Colombia: Socioeconomic and Financial Determinants. Washington. Inter-American Development Bank, .
CONPEs (2002). Bases de la politica de vivienda 20022006: ajustes al programa de subsidio familiar de vivienda e incentivos de oferta y demanda para créditos de vivienda en UVRs - Documento \# 3200. Bogotá, D.C.: Departamento Nacional de Planeación.

Cowen, M. y Shenton, R. (1996). Doctrines of Development. London: Routledge.

Datta, K. (2009). Transforming South-North relations? International migration and development. Geography Compass, 3 (1), 108-134.

Datta, K. y Jones, G. A. (2001). Housing and finance in developing countries: invisible issues on research and policy agendas. Habitat International, 25, 333-357.

De Haas, H. (2012). The Migration and Development Pendulum: A Critical View on Research and Policy. International Migration, 50 (3), 8-25.

Delgado-Wise, R. y Márquez Covarrubias, H. (2010). Understanding the Relationship between Migration and Development: Toward a New Theoretical Approach. In Glick Schiller, N. y Faist, T. (eds.). Migration, Development and Transnationalization: A Critical Stance. New York: Berghahn Books.

Delgado-Wise, R., Márquez Covarrubias, H. y Puentes, R. (2013). Reframing the debate on migration, development and human rights. Population, Space and Place, 19 (4), 430-442.

Dinero (2011a). Locomotora de la construcción ya despegó y va volando, 11/3/11.

Dinero (2011b). La locomotora en marcha, 13/10/11. El Espectador (2010a). Santos reconoce que los colombianos prefieren el "gota a gota" y no un banco, 27/8/10.

El Espectador (2010b). "Vamos a construir un millón de viviendas", 4/12/10. 
Escobar, A. (1989). The professionalization and institutionalization of "development" in Colombia in the early post-world war II period. International Journal of Educational Development, 9 (2), 139-154.

Escobar, A. (1995). Encountering Development: the making and unmaking of the Third World. New Jersey: Princeton University Press.

Faist, T. (2008). Migrants as transnational development agents: an inquiry into the newest round of the migration-development nexus. Population, Space and Place, 14, 21-42.

Fajnzylber, P. y López, H. (2007). Close to Home: The Development Impact of Remittances in Latin America. Washington, D.C.: The International Bank for Reconstruction and Development / The World Bank.

Gamlen, A. (2010). The New Migration and Development Optimism: A Review of the 2009 Human Development Report. Global Governance, 16, 415-422.

Gamlen, A. (2014). The new migration-and-development pessimism. Progress in Human Geography, 38 (4), 581-597.

Gamlen, A. y Marsh, K. (2011). Introduction: Modes of governing global migration. En Gamlen, A. y Marsh, K. (eds.). Migration and Global Governance (pp. 1-33). London: Edward Elgar.

Garay, L. J. y Rodríguez, A. (2005). La migración internacional: una sintesis de aproximaciones teóricas alternativas. Bogotá D.C.: Organización Internacional para las Migraciones (oIM).

Geiger, M. y Pécoud, A. (2013). Special Issue: Migration, Development and the "Migration and Development Nexus". Population, Space and Place, 19 (4), 369-374.

Gilbert, A. (1987). Latin America's urban poor: shanty dwellers or renters of rooms? Cities, 4, 43-51.
Gilbert, A. (1997). On subsidies and home-ownership: Colombian housing policy during the 1990s. Third World Planning Review, 19 (1), 51-69.

Gilbert, A. (1998). The Latin American City. Nottingham: Latin American Bureau.

Gilbert, A. (2004). Helping the poor through housing subsidies: lessons from Chile, Colombia and South Africa. Habitat International, 28, 13-40.

Gilbert, A. (2008). Slums, tenants and home-ownership: on blindness to the obvious. International Development Planning Review, 30 (2), i-x.

Gilbert, A. y Varley, A. (1991). Landlord and tenant: housing the poor in urban Mexico. London: Routledge.

Gilbert, A. y Ward, P. M. (1982). Low-income housing and the State. En Gilbert, A., Hardoy, J. E. y Ramírez, R. (eds.). Urbanization in Contemporary Latin America (pp. 79-127). New York / Chichester, England: John Wiley \& Sons Ltd.

Gilbert, A. y Ward, P. M. (1985). Housing, the state and the poor: policy and practice in three Latin American cities. Cambridge: Cambridge University Press.

Gómez, M., Bougher, L. y Robertson, I. (2005). Making remittances work for development: leveraging remittances to the housing market. New York: Columbia University - School of International and Public Affairs.

Gough, K. V. (1999). Affording a home: the strategies of self-help builders in Colombia. En Datta, K. y Jones, G. A. (eds.). Housing and Finance in Developing Countries (pp. 119-135). London: Routledge.

Guarnizo, L. E. (2006). El Estado y la migración global colombiana. Migración y desarrollo (006), 79-101.

Guarnizo, L. E. (2008). Londres latina: la presencia colombiana en la capital británica. México, D.F.: Miguel Ángel Porrúa. 
Guarnizo, L. E., Sánchez, A. I. y Roach, E. M. (1999). Mistrust, fragmented solidarity, and transnational migration: Colombians in New York and Los Angeles. Ethnic and Racial Studies, 22 (2), 367-396.

Hall, J. (2010). Diez años de innovación en remesas: lecciones aprendidas y modelos para el futuro. Washington, D.C.: Banco Interamericano de Desarrollo.

Jaramillo, M. A. y Mejía, W. (2008). Situaciones más relevantes que dificultan el proceso de adquisición de vivienda en Colombia por parte de los emigrantes internacionales. Bogotá: Alma Mater - Red de Universidades Públicas del Eje Cafetero.

Jones, G. A. y Datta, K. (1999). From self-help to selffinance: the changing focus of urban research and policy. En Datta, K. y Jones, G. A. (eds.). Housing and Finance in Developing Countries (pp. 3-25). London: Routledge

Jones, G. A. y Ward, P. M. (1994a). Tilting at windmills: paradigm shifts in World Bank orthodoxy. En Jones, G. A. y Ward, P. M. (eds.). Methodology for Land and Housing Market Analysis (pp. 8-23). London: UCL Press.

Jones, G. A. y Ward, P. M. (1994b). The World Bank's "New" Urban Management Programme: Paradigm Shift or Policy Continuity? Habitat International, 18 (3), 33-51.

Klaufus, C. (2010a). The two ABCs of aided self-help housing in Ecuador. Habitat International, 34, 351-358.

Klaufus, C. (2010b). Watching the city grow: remittances and sprawl in intermediate Central American cities. Environment and Urbanization, 22 (1), 125-137.

Levitt, P. (2001b). Transnational migration: taking stock and future directions. Global Networks, 1 (3), 195-216.
Mcllwaine, C., Cock, J. C. y Linneker, B. (2011). No Longer Invisible: the Latin American community in London. London: Queen Mary, University of London.

Ministerio del Interior y de Justicia (2009). Decreto 1143 de 2009. Recuperado de http://www.mij. gov.co/normas/2009/d11432009.htm.

Ministerio de Relaciones Exteriores (2004). Colombia Nos Une (promotional brochure). Bogotá: Ministerio de Relaciones Exteriores.

Peck, J. y Theodore, N. (2010). Mobilizing policy: Models, methods and mutations. Geoforum, $41,169-174$.

Peck, J. y Tickell, A. (2002). Neoliberalizing Space. Antipode, 34 (3), 380-404.

PND (2010). Plan Nacional de Desarrollo 2010-2014 - Prosperidad para Todos. Bogotá: Imprenta Nacional.

Portes, A. (2003). Theoretical Convergencies and Empirical Evidence in the Study of Immigrant Transnationalism. International Migration Review, 37 (3), 874-892.

Portes, A. (2009). Migration and development: reconciling opposite views. Ethnic and Racial Studies, 32 (1), 5-22.

Roy, A. (2010). Poverty Capital: Microfinance and the Making of Development. New York: Routledge.

Saldaña-Portillo, M. J. (2003). The Revolutionary Imagination in the Americas and the Age of Development. Durham: Duke University Press.

Smith, M. P. (1988). City, State \& Market: The Political Economy of Urban Society. Oxford: Blackwell.

Smith, S. J. (2008). Owner-occupation: at home with a hybrid of money and materials. Environment and Planning A, 40 (3), 520-535.

Smith, S. J., Easterlow, D. y Munro, M. (2004). Housing for health: does the market work? Environment and Planning A, 36 (4), 579-600. 
Smith, S. J., Munro, M. y Christie, H. (2006). Performing (Housing) Markets. Urban Studies, 43 (1), 81-98.

UN-Habitat (2005). Financing Urban Shelter. London: Earthscan.

UN-Habitat (2012). Global Urban Indicators. Recuperado de http://www.unhabitat.org/stats/ Default.aspx.

undP (2010). Human Development Report 2009, Overcoming Barriers: Human Mobility and Development. New York: UNDP.

Varley, A. (1998). The Political Uses of Illegality: Evidence from Urban Mexico. En Fernandes, E. y Varley, A. (eds.). Illegal Cities: Law and Urban
Change in Developing Countries (pp. 172-190). London: Zed Books.

Winchester, L. (2005). Sustainable human settlements development in Latin America and the Caribbean. Santiago: Cepal - Sustainable Development and Human Settlements Division.

World Bank (2006). The Development Impact of Workers' Remittances in Latin America Report 37026. Washington, D.C.: World Bank.

World Bank (2011). Migration and Remittances Factbook 2011. Washington D.C.: World Bank.

Zapata, G. P. (2013). The Migration-Development Nexus: Rendering Migrants as Transnational Financial Subjects through Housing. Geoforum, 47, 93-102. 